\title{
Comparison of add-on efficacies of formoterol and tulobuterol on budesonide inhalation in elderly patients with asthma
}

\author{
Susumu Fukahori ${ }^{1}$, Tetsuya Kawano ${ }^{1}$, YASUSHI OBASE ${ }^{1}$, Jun Iriki ${ }^{1}$, Tomoko \\ Tsuchida-Yabe ${ }^{1}$, Shinya Tomari ${ }^{2}$, Chizu Fukushima ${ }^{1}$, Hiroto Matsuse ${ }^{3}$, and Hiroshi Mukae ${ }^{1}$ \\ ${ }^{1}$ Nagasaki University Hospital \\ ${ }^{2}$ Isahaya General Hospital, Japan Community Health care Organization (JCHO) \\ ${ }^{3}$ Toho University Ohashi Medical Center
}

July 7, 2020

\begin{abstract}
Rationale, aims and objectives; For asthma strategy, to avoid the aggravation of bronchial inflammation and contraction, the addition of long acting beta agonist (LABA) to inhaled corticosteroids (ICS) has been recommended. To know whether there is any clinical difference between the additional efficacies of formoterol (FOR) and tulobuterol (TUL) onto budesonide (BUD) may be useful for the elderly patients' asthma treatment strategy. Method: Eighteen outpatients with mild to moderate bronchial asthma with FEV1.0\% < 80\% treated by intermediate ICS dosages visited Respiratory Division of Nagasaki University Hospital or Isahaya General Hospital, Japan Community Health care Organization were subjected. Subjects were randomly assigned (9 cases per group) to either the FBC group (BUD/FOR 160/4.5 mcg: 2 inhalations twice daily) or BUD + TUL group (BUD 200 mcg: 2 inhalations twice daily + TUL $2 \mathrm{mg}$ daily) and were compared in parallel with 2 arms for 12 weeks prospectively. PEF, FEV1, impulse oscillometry (IOS), fractional exhaled nitric oxide, Asthma Control Questionnaire and mini-Asthma Quality of Life Questionnaire (mini-AQLQ), and occurrence of adverse reactions were compared. Results; The "Fres" of IOS was improved $(\mathrm{p}=0.03)$ in FBC group. The "emotion" domain of mini-AQLQ was improved $(\mathrm{p}=0.03)$ in BUD+TUL group. Conclusions; By changing the drug formulation, the patch was superior in terms of satisfaction, but it was thought that the inhaled combination was superior in improving the respiratory function itself. It is necessary to pay attention to the characteristics of the patient when selecting treatment.
\end{abstract}

Comparison add-on efficacies of formoterol and tulobuterol on budesonide inhalation in elderly patients with asthma

\section{Short title}

Additional effects of Formoterol or Tulobuterol on budesonide for elderly with asthma

Declarations of interest

The authors declare that they have no conflict of interest.

Abstract (232 words)

For asthma strategy, to avoid the aggravation of bronchial inflammation and contraction, the long acting beta agonist (LABA) addition on inhaled corticosteroids (ICS) has been recommended. To know whether there is any clinical difference between the additional efficacies of formoterol (FOR) and tulobuterol (TUL) onto budesonide (BUD) may be useful for the elderly patients' asthma treatment strategy. 18 outpatients with mild to moderate bronchial asthma with FEV1.0\% $<80 \%$ treated by intermediate ICS dosages visited Respiratory Division of Nagasaki University Hospital or Isahaya General Hospital, Japan Community Health 
care Organization were subjected, and were randomly assigned (9 cases per group) to either the FBC group (BUD/FOR 160/4.5 $\mu \mathrm{g}, 2$ inhalations twice daily) or BUD + TUL group (BUD $200 \mathrm{mcg}$ : 2 inhalations twice daily + TUL $2 \mathrm{mg}$ daily) and were compared in parallel with 2 arms for 12 weeks prospectively. Peak expiratory flow, forced expiratory volume in 1 second, impulse oscillometry (IOS), fractional exhaled nitric oxide (FeNO), Asthma Control Questionnaire, mini-Asthma Quality of Life Questionnaire (mini-AQLQ), and occurrence of adverse reactions were compared. The "Fres" of IOS was improved in FBC group ( $\mathrm{p}=0.03)$. The "emotion" domain of mini-AQLQ was improved in BUD+TUL group $(\mathrm{p}=0.03)$. By changing the drug formulation, the patch was superior in terms of satisfaction, but it was thought that the inhaled combination was superior in improving the respiratory function itself. It is necessary to pay attention to the characteristics of the patient when selecting treatment.

Keywords

bronchial inflammation, forced expiratory volume in 1 second (FEV1), fractional exhaled nitric oxide (FeNO), impulse oscillometry (IOS), mini-Asthma Quality of Life Questionnaire (mini-AQLQ), prospective study, transdermal delivery system

Introduction

Because the essential pathology of bronchial asthma is bronchial inflammation and airway smooth muscle contraction, inadequate treatments result in increased airway hyperreactivity and future exacerbations due to remodeling and/or smooth muscle hypertrophy $(1,2)$. The long acting beta agonist (LABA) addition on inhaled corticosteroids (ICS) has been recommended in Step2 onward to treat the bronchial inflammation and contraction. Though ICS and LABA combination inhalation therapy has an advantage as these materials goes to almost same area in the airways $(3,4)$, of which effects would be insufficient without adequate inhalation technique, e.g. elder patients with lower respiratory function. Even for such patients, the use of systemic steroids as a substitute for ICS should be avoided in terms of adverse effects (5). Regarding LABA, the tulobuterol patch (Hokunalin ${ }^{\mathrm{TM}}$ Tape; TUL) with the transdermal delivery system has been used for control of asthma and chronic obstructive pulmonary disease (COPD) in Japan, Korea, and China (6). This patch prevents excessive increase of the concentration in blood that is useful to reduce the systemic adverse reactions $(7,8)$.

There are several studies reported the additional efficacies of TUL and salmeterol (SAL) onto fluticasone propionate (FLU) inhalation (9-11), and the results were inconsistent. There are little studies compared the additional efficacies of TUL and formoterol (FOR), an LABA inhalation onto budesonide (BUD) inhalation, an ICS. Because FOR/BUD combination inhalation is quite popular for asthma control and BUD is known as an ICS with less side effects $(3,4)$, to know any clinical difference between the additional impacts efficacies of FOR and TUL onto BUD may be useful for the elderly patients' asthma treatment strategy. We compared these strategies by a randomized prospective study with 2 arms for 12 weeks.

Methods

Subjects

In this study, 18 elderly outpatients 65 years of age or older, with mild to moderate bronchial asthma, with FEV1.0\% $<80 \%$ treated by intermediate ICS dosages visited Respiratory Division of Nagasaki University Hospital or Isahaya General Hospital, Japan Community Health care Organization (JCHO) were recruited (Table 1). All included patients fulfilled the Global Initiative for Asthma (GINA) criteria (1), and had a history of asthmatic symptoms, including cough, wheezing, or dyspnea (mean (SD) age was 72.8 (6.2), female $/$ male $=9 / 9$, Non-smoker $/$ Ex-smoker $/$ Current smoker $=7 / 11 / 0)$. All patients had no findings of COPD on Chest X ray or high resolution CT. All patients retained normal diffusion capacity. Antiasthma drugs were discontinued for at least $24 \mathrm{~h}$ prior to each examination. The intermediate dosages of ICS were defined as follows: 1) SAL/FUL combination, 200 to $500 \mu \mathrm{g} /$ day or 2) BUD, 400 to $800 \mu \mathrm{g} /$ day; becromethason, FUL, cicresonide, or mometasone, 200 to $400 \mu \mathrm{g} /$ day. The ethics committee of Nagasaki University Hospital approved this study protocol (\#11042549), and all the participants received verbal and 
written information and provided the informed consent.

Study protocol

Subjects were randomly assigned to either the FBC group $(\mathrm{n}=9$, BUD/FOR, 160/4.5 $\mu \mathrm{g}, 2$ inhalations twice daily) or BUD + TUL group ( $\mathrm{n}=9$, BUD $200 \mu \mathrm{g}, 2$ inhalations twice daily + TUL 2 mg daily) and these groups were compared. The treatment period was 12 weeks and the BUD dose of both groups were set as equal. The short-acting inhaled $\beta 2$-agonists (SABA) was permitted to be used as needed (Fig1).

At the start of the study, a full medical interview was given and a physical examination was performed. No patients had abnormal electrocardiogram findings. During a 2 week run-in period, they were asked to keep a daily diary card. This card was for record morning and evening peak expiratory flow (PEF), symptom score and the rescue use of SABA inhaler.

Measurements

PEF, forced expiratory volume in 1 second (FEV1), impulse oscillometry (IOS), fractional exhaled nitric oxide (FeNO), Asthma Control Questionnaire (ACQ) and mini-Asthma Quality of Life Questionnaire (miniAQLQ), and occurrence of adverse reactions were observed.

Pulmonary function test

Spirometry was measured using a Chestac-8900 (Chest Co., Ltd., Japan). For the predicted values of FEV 1 and vital capacity (VC), the reference data developed by the Japanese Respiratory Society were taken as the standard value (12).

Forced oscillation technique

The factors of IOS were measured using a forced oscillation technique device (MostGraph-01; Chest Co., Ltd,) (13). During tidal breath about 60 seconds in the sitting position, respiratory impedance were measured. To reduce upper airway shunting, the subject's cheeks and mouth floor were supported by the patient's both hands. The levels of Rrs at $5 \mathrm{~Hz}$ (R5), Rrs at $20 \mathrm{~Hz}$ (R20), the difference between R5 and R20 (R5-R20), Xrs at $5 \mathrm{~Hz}$ (X5), resonant frequency (Fres), and also the differences of the mean Rrs and Xrs in the expiratory phase to those in the inspiratory phase were evaluated. Whole-breath analysis and within-breath analysis were performed by programmed software automatically. These forced oscillation technique measurements were performed prior to other pulmonary function tests.

FeNO

FeNO was measured by portable sensor (NIOX MINO; Aerocrine AB, Solna, Sweden) according to ATS/ERS recommendations on measurements of FeNO (14), and the results were expressed as parts per billion. The participants were asked to inhale through the device and exhale steadily for 10 seconds at a flow rate of 50 $\mathrm{ml} / \mathrm{s}$ and at a pressure of $10 \mathrm{~cm} \mathrm{H}_{2} \mathrm{O}$. The measurements were done in a sitting position.

ACQ and mini-AQLQ

ACQ is a survey for asthma control contains seven items about limitation due to asthma measured on a 7-point scale, from 0 (no impairment) to 6 (extreme impairment), using the past 7 days recall. The mean score $<=0.75$ was classified as "well controlled", $>=1.5$ as "uncontrolled", and between these points as "somewhat controlled". A Minimal Clinically Important Difference (MCID) of 0.5 was used (15).

Mini-AQLQ is simplified version with 15 questions with four domains (symptoms, activities, emotions, and environment) from original AQLQ with 32 questions, and ask each question to have "1" if there is a serious impairment and "7" if there is no problem at all. The mean score of [?]1.5 indicates uncontrolled asthma and $<1.5$ indicates well controlled asthma. The MCID is a mean change in this mean score of greater than $0.5(16)$.

Evaluation of the treatment effects 
The changes of the each factor ( $\Delta$ factor) was defined by subtracking the value of Visit 1 from that of Visit 4 .

Statistical analysis

Mann-Whitney test was used for comparison of the background factors and the changes of the factors $(\Delta$ factor) between the groups. The p-value $<0.05$ was considered as significant difference.

Results

Patient demographics

Regarding age, sex, classification of disease (atopic or non-atopic), severity, ACQ, AQLQ, spirometry, FeNO, and IOS parameters, there were no significant differences between the groups (Table 1).

The changes of parameters in ACQ, AQLQ, spirometry, FeNO, and IOS

3 cases ( 1 of FBC group and 2 of BUD+TUL group) had to use systemic steroids within 2 weeks after the commencement of the study and discontinued the trial. The remaining 15 cases were subjected for efficacy analysis. The "Fres" of IOS was improved $(\mathrm{p}=0.03)$ in FBC group. The "emotion" domain of AQLQ was improved $(\mathrm{p}=0.03)$ in BUD+TUL group (Table 2). There were no differences between the groups in the changes of each factor of ACQ, spirometry, or FeNO.

Adverse events

No drug-related adverse reactions were noted in both groups.

Discussion

In this study, FBC group had better improvement on "Fres" of IOS indicators, and BUF+TUL group had better improvement on "emotion" domain of mini-AQLQ. It may be suggested the characteristics of the additional effects of each drug (FOR and TUL) differ.

By adding FOR to BUD (equal to FBC inhalation), the "Fres" associated with large and small airway resistance was improved significantly $(\mathrm{p}=0.03)$. FOR is a LABA and provides sustained action for 12 hours which needs to be inhaled twice a day (17). It is reasonable for most patients who can inhale the medicine properly, the ICS/LABA combination will be suitable for asthma control (18).

In contrast, adding TUL to BUD improved "emotion" domain significantly $(\mathrm{p}=0.03)$. Previously, the improvement of asthma control status of patients with adult-onset mild to moderate asthma by additional TUL on FLU inhalation was reported (9), and additional effect of TUL on leukotriene receptor antagonist in children with asthma was also reported (10). The efficacy of TUL addition to BUD inhalation was firstly found by this study.

Other studies, however, reported that SAL addition achieved better control after switching from TUL addition in asthmatic patients with FLU treatment (10), or that SAL addition on FLU improved morning and evening PEF rates and AQLQ score but TUL addition did not, in patients with asthma on ICS therapy in 8 weeks observation (11). In our study, we have found the addition of TUL or FOR on BUD respectively showed characteristic improvements on "Fres" or "mini-AQLQ".

For the patients with typical asthma, respiratory functions are most severely suppressed from midnight to early morning, and it is called as morning dip (19). Suppression of this morning dip may improve the patient's QOL. The therapeutic adherence of the TUL patch was significantly higher than that of other inhaled drugs, as the patch was easy to use and was applied once a day (6). TUL patch prolongs the drug's action to 24 hours, which may improve the circadian rhythm $(8,20)$. Improvements of QOL or treatment adherence are very important point for chronic disease including asthma (21). Regarding the change in FeNO, there was no difference between the groups, and the BUD dose was consistently the same in both groups, so this was understandable. 
In the study, there are some limitations. Firstly, the patients were recruited from the asthma specialist outpatient department, which might induce any bias compared with general medical clinics, and the numbers were very small. Secondly, because the TUL may suppress the Rhinovirus infection (22) and may be effective on upper respiratory tract infection symptoms (23), the setting period include the winter season and for at least half year might be desired.

In conclusion, by changing the drug formulation, the patch was superior in terms of patients' satisfaction, but it was thought that the inhaled combination was superior in improving the respiratory function itself. It is necessary to pay attention to the characteristics of the patient when selecting treatment.

Acknowledgement

We would like to thank the physicians, patients, and volunteers for participating in this study. This research was supported by a research grant from the Non-profit Organization Aimed to Support Community Medicine Research in Nagasaki.

References

1. Global Initiative for Asthma (GINA). Global strategy for asthma management and prevention. NHLBI/WHO Report. Bethesda National Institutes of Health, National Heart, Lung and Blood Institute. NIH Publication: 02-3659. Updated 2015. Available from URL:http://www.ginasthma.org.

2. Ichinose M, Sugiura H, Nagase H, Yamaguchi M, Inoue H, Sagara H, Tamaoki J, Tohda Y, Munakata M, Yamauchi K, Ohta K; Japanese Society of Allergology. Japan asthma guideline Japanese guidelines for adult asthma 2017. Allergol Int. 2017;66:163-189.

3. Patel M, Pilcher J, Pritchard A, Perrin K, Travers J, Shaw D, Holt S, Harwood M, Black P, Weatherall M, et al. SMART Study Group. Efficacy and safety of maintenance and reliever combination budesonide-formoterol inhaler in patients with asthma at risk of severe exacerbations: a randomised controlled trial. Lancet Respir Med. 2013;1:32-42.

4. Sobieraj DM, Weeda ER, Nguyen E, Coleman CI, White CM, Lazarus SC, Blake KV, Lang JE, Baker WL. Association of Inhaled Corticosteroids and Long-Acting $\beta$-Agonists as Controller and Quick Relief Therapy With Exacerbations and Symptom Control in Persistent Asthma: A Systematic Review and Meta-analysis. JAMA. 2018;319:1485-1496.

5. Kawano T, Matsuse H, Obase Y, Kondo Y, Machida I, Tomari S, Mitsuta K, Fukushima C, Shimoda T, Kohno S. Hypogammaglobulinemia in steroid-dependent asthmatics correlates with the daily dose of oral prednisolone. Int Arch Allergy Immunol. 2002;128:240-243.

6. Tamura G, Ichinose M, Fukuchi Y, Miyamoto T. Transdermal tulobuterol patch, a long-acting $\beta(2)-$ agonist. Allergol Int. 2012;61:219-229.

7. Park SI, Kim BH. Bioequivalence assessment of tulobuterol transdermal delivery system in healthy subjects. Int J Clin Pharmacol Ther. 2018;56:381-386.

8. Iikura Y, Uchiyama H, Akimoto K, Ebisawa M, Sakaguchi N, Tsubaki T, Ishizu H, Kabayama H, Yagi $\mathrm{K}$, Miura K, et al. Pharmacokinetics and pharmacodynamics of the tulobuterol patch, HN-078, in childhood asthma. Ann Allergy Asthma Immunol. 1995;74:147-151.

9. Inoue H, Niimi A, Matsumoto H, Ito I, Oguma T, Otsuka K, Takeda T, Nakaji H, Tajiri T, Iwata T,et.al. A 12-week, randomized, parallel-group, proof-of-concept study of tulobuterol patch and salmeterol inhaler as add-on therapy in adult-onset mild-to-moderate asthma. Clin Exp Pharmacol Physiol. 2017;44:21-29.

10. Kobayashi Y, Yasuba H, Kudou M, Kita H. Addition of transdermal or inhaled long-acting Beta2agonists in adult asthmatic patients treated with inhaled corticosteroids: switchover study from tulobuterol patch to salmeterol dry powder inhaler. J Asthma. 2007;44:77-81.

11. Fujimoto K, Komatsu Y, Yasuo M, Urushihata K, Kubo K. Comparison of the clinical efficacy of salmeterol and sustained-release tulobuterol (patch) on inadequately controlled asthma patients on inhaled corticosteroids. J Asthma. 2006;43:501-507.

12. Sasaki E, Nakamura M, Kida K. Reference values for spirogram and blood gas analysis in Japanese adults. J Jpn Respir Soc. 2001;39:S1-S17. 
13. Shirai T, Kurosawa H. Clinical application of the forced oscillation technique. Intern Med. 2016;55:559566.

14. ATS/ERS. ATS/ERS recommendations for standardized procedures for the online and offline measurement of exhaled lower respiratory nitric oxide and nasal nitric oxide, 2005. American J Resp and Crit Care Med. 2005;171:912-930.

15. Juniper EF, O'Byrne PM, Guyatt GH, Ferrie PJ, King DR. Development and validation of a questionnaire to measure asthma control. Eur Respir J 1999;14:902-907.

16. Juniper EF, Guyatt GH, Cox FM, Ferrie PJ, King DR. Development and validation of the Mini Asthma Quality of Life Questionnaire. Eur Respir J. 1999;14:32-38.

17. Janjua S, Schmidt S, Ferrer M, Cates CJ. Inhaled steroids with and without regular formoterol for asthma: serious adverse events. Cochrane Database Syst Rev.2019;9: pages not shown.

18. Masoli M, Weatherall M, Holt S, Beasley R. Moderate dose inhaled corticosteroids plus salmeterol versus higher doses of inhaled corticosteroids in symptomatic asthma. Thorax. 2005;60:730-734.

19. Turner-Warwick M. On observing patterns of airflow obstruction in chronic asthma. Br J Dis Chest 1977;71:73-86.

20. Patel KR. Tulobuterol in the management of obstructive airways disease in adults. Clin Ther. 1985;7:452-467.

21. O'Byrne PM, Jenkins C, Bateman ED. The paradoxes of asthma management: time for a new approach? Eur Respir J. 2017;50: pages not shown.

22. Yamaya M, Nishimura H, Nadine L, Kubo H, Ryoichi N. Tulobuterol inhibits rhinovirus infection in primary cultures of human tracheal epithelial cells. Physiol Rep. 2013;1:pages not shown

23. Katsunuma T, Ohya Y, Fujisawa T, Akashi K, Imamura N, Ebisawa M, Daikoku K, Kondo N, Terada A, Doi S, et al. Protective Effect of Tulobuterol Patch on the Long-term Management of Asthma in Young Children Study Group.Effects of the tulobuterol patch on the treatment of acute asthma exacerbations in young children. Allergy Asthma Proc. 2012;33:e28-34.

\section{FBC group}

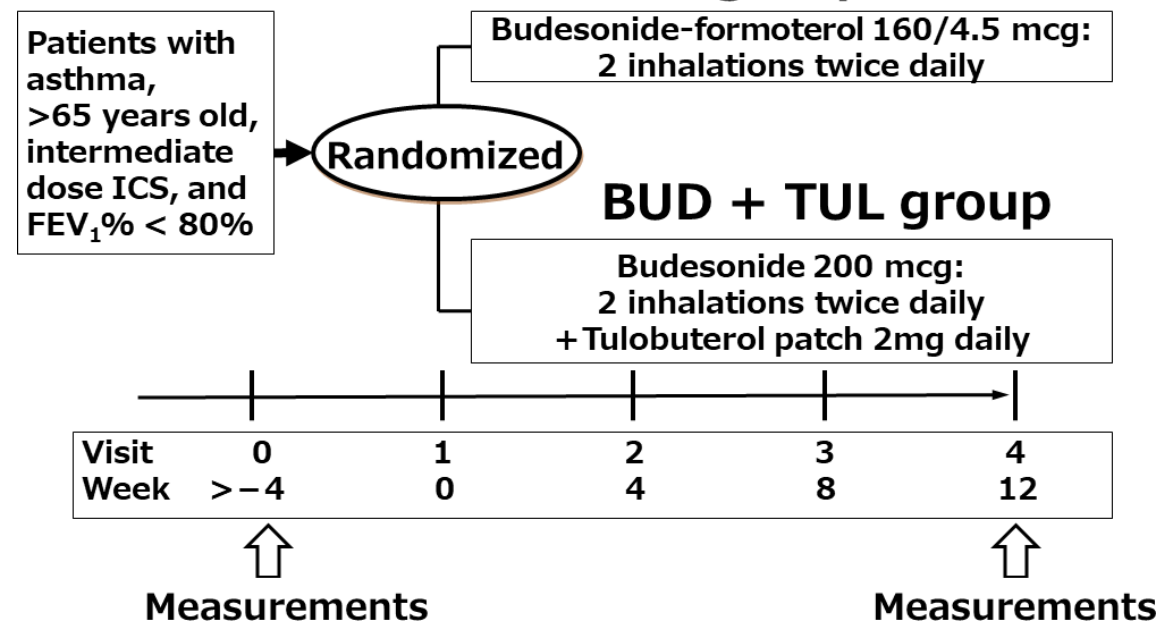

\section{Hosted file}

Differences of add-on FOL or TUL on BUD Table 1.docx available at https://authorea.com/users/ 339460/articles/465668-comparison-of-add-on-efficacies-of-formoterol-and-tulobuterol-onbudesonide-inhalation-in-elderly-patients-with-asthma

\section{Hosted file}

Differences of add-on FOL or TUL on BUD Table 2.docx available at https://authorea.com/users/ 
339460/articles/465668-comparison-of-add-on-efficacies-of-formoterol-and-tulobuterol-onbudesonide-inhalation-in-elderly-patients-with-asthma 